\title{
Fechamento de fístula bucossinusal com gordura de Bichat: relato de caso
}

Bucosinusal fistula closure with Bichat's fat: case report

Cierre de fístula bucosinusal con bola adiposa de Bichat: reporte de caso

Brenda Karoline Valença FREIRE ${ }^{1}$

Henrique Lima Ferreira de SOUZA ${ }^{1}$

Heitor Ferreira de SOUZA NETO ${ }^{1}$

Éwerton Daniel Rocha RODRIGUES ${ }^{2}$

Renata de Albuquerque Cavalcanti ALMEIDA ${ }^{3}$

Emerson Filipe de Carvalho NOGUEIRA ${ }^{4}$

'Curso de Graduação em Odontologia no Centro Universitário Brasileiro (UNIBRA),50050-230 Recife - PE, Brasil

${ }^{2}$ Especialista e Mestre em Cirurgia e Traumatologia Bucomaxilofacial, Professor de Implantodontia no Centro Universitário Brasileiro (UNIBRA) 50050-230 Recife - PE, Brasil

${ }^{3}$ Especialista, Mestre e Doutora em Cirurgia e Traumatologia Bucomaxilofacial, Professora de Cirurgia Oral, Centro Universitário Brasileiro (UNIBRA), 50050-230 Recife - PE, Brasil

${ }^{4}$ Especialista e Mestre em Cirurgia e Traumatologia Bucomaxilofacial. Professor de Cirurgia Oral, Centro Universitário Brasileiro (UNIBRA), 50050-230 Recife - PE, Brasil

\section{Resumo}

Introdução: A gordura de Bichat consiste num tecido gorduroso amplamente utilizado para correção de complicações cirúrgicas e defeitos ósseos. Seu uso se dá pela rica vascularização sanguínea, menor morbidade ao sítio doador, rápida epitelização, altas taxas de sucesso, confiabilidade e por não causar sequelas estéticas visíveis. Objetivo: Relatar um caso clínico de um paciente que apresentou comunicação bucossinusal, em que foi utilizada a gordura de Bichat para o seu tratamento. Relato do caso: Paciente do sexo feminino, 56 anos de idade, apresentou queixa de dor em face e saída de líquidos em cavidade nasal. Ao exame tomográfico foi observada imagem sugestiva de perda óssea em soalho do seio maxilar com velamento local. $O$ tratamento consistiu na fistulectomia, e reposicionamento da gordura de Bichat sobre o defeito ósseo, com posterior manutenção do retalho por sutura com fio de seda 4-0, sob anestesia local. A paciente foi acompanhada por 12 meses sem sinais de recidiva. Conclusão: A utilização da gordura de Bichat para o fechamento da comunicação bucossinusal é uma modalidade de tratamento simples, eficaz e confiável, apresentando grandes vantagens quando comparada à outras técnicas.

Descritores: Corpo Adiposo; Fístula Bucal; Sinusite Maxilar.

\section{Abstract}

Introduction: Bichat's fat consists of a fatty tissue widely used to correct surgical complications and bone defects. Its use is due to the rich blood vascularity, less morbidity to the donor site, rapid epithelialization, high success rates, reliability and for not causing visible aesthetic sequelae. Objective: Report a clinical case of a patient who presented oroantral communication, in which Bichat's fat was used for its treatment. Case report: A 56-year-old female patient complained of pain in the face and fluid left in the nasal cavity. On tomographic examination, an image suggestive of bone loss was observed on the floor of the maxillary sinus with local veiling. The treatment consisted of fistulectomy, apprehension and repositioning of Bichat's fat on the bone defect, with subsequent maintenance of the flap by suturing with 4-0 silk thread, under local anesthesia. The patient was accompanied for 12 months with no signs of recurrence. Conclusion: The use of Bichat fat to close oroantral communication is a simple, effective and reliable treatment modality, with great advantages when compared to other techniques.

Descriptors: Fat Body; Oral Fistula; Maxillary Sinusites.

\section{Resumen}

Introducción: La grasa de Bichat consiste en un tejido graso muy utilizado para corregir complicaciones quirúrgicas y defectos óseos. Su uso se debe a la rica vascularización sanguínea, menor morbilidad en el sitio donante, rápida epitelización, altas tasas de éxito, confiabilidad y por no causar secuelas estéticas visibles. Objetivo: Informar un caso clínico de un paciente que presentó comunicación del seno bucal, en el que se utilizó grasa de Bichat para su tratamiento. Caso clínico: paciente de 56 años que se quejaba de dolor en la cara y fuga de líquido por la cavidad nasal. En el examen tomográfico se observó una imagen sugestiva de pérdida ósea en el suelo del seno maxilar con velo local. El tratamiento consistió en fistulectomía y reposicionamiento de la grasa de Bichat sobre el defecto óseo, con posterior mantenimiento del colgajo mediante sutura con hilo de seda 4-0, bajo anestesia local. El paciente fue seguido durante 12 meses sin signos de recurrencia. Conclusión: El uso de Bichat fat para la comunicación oral cercana es una modalidad de tratamiento simple, eficaz y confiable, con grandes ventajas frente a otras técnicas.

Descriptores: Cuerpo Adiposo; Fistula Oral; Sinusitis Maxilar.

INTRODUÇÃO

Os seios maxilares são cavidades
aéreas, nos ossos maxilares, revestidas por mucosa. Por apresentar, anatomicamente, relação estreita com os ápices radiculares dos elementos dentários superiores posteriores, procedimentos de exodontias podem estabelecer uma comunicação entre a cavidade bucal e os seios maxilares, conhecida como comunicação bucossinusal $^{1,2}$.

Quando estabelecida a comunicação, o tratamento cirúrgico será necessário em casos onde haja quaisquer sinais de inflamação, infecção do seio maxilar e/ou uma comunicação com diâmetro maior que $3 \mathrm{~mm}$. Nesses casos, seu fechamento pode ser realizado através de retalhos mucosos locais, materiais aloplásticos, como enxertos ósseos autógenos, velamento por malha de titânio associada a retalho mucoperiosteal, enxerto de folhas de ouro, enxertos combinados com regeneração óssea e regeneração tecidual guiada, transplante de terceiros molares naturais, blocos de hidroxiapatita não porosos e o recobrimento do defeito ósseo com a gordura de Bichat ${ }^{1,3,4,5}$.

A utilização da gordura de Bichat para a correção do defeito osséo é uma técnica simples e de fácil acesso, com alta confiabilidade por apresentar rica vascularização sanguínea, quando comparada à outras técnicas, apresenta menor morbidade ao 
sítio doador, rápida epitelização, altas taxas de sucesso descritas na literatura e por não deixar cicatrizes visíveis por ser tratar de um procedimento realizado intraoral. A técnica para coleta da gordura de Bichat consiste na realização do acesso cirúrgico com incisão no periósteo, em região posterior ao pilar zigomático, posteriormente, é realizada uma minuciosa dissecção, medial ao processo coronóide, evidenciando o tecido adiposo com o auxílio de pinças delicadas, até sua liberação e avanço do retalho ${ }^{6}$.

O objetivo deste trabalho é relatar um caso de fechamento de fístula bucossinusal com a gordura de Bichat.

CASO CLÍNICO

Paciente do sexo masculino, 49 anos de idade, compareceu ao ambulatório queixa de dor em face, cefaleia constante e halitose. $O$ mesmo apresentava história de remoção de elemento dentário superior há 4 meses, o qual foi o ponto inicial para o desenvolvimento dos sintomas, bem como pela saída de líquidos pelo nariz quando ingeria algo. $O$ otorrinolaringologista o avaliou e diagnosticou a presença de sinusite em seio maxilar e frontal, onde o quadro agudo foi controlado com uso de amoxicilina associado ao clavulanato de potássio, além do uso de corticoide tópico por via nasal por 15 dias.

Ao exame físico observou-se presença de uma depressão em rebordo alveolar na região do elemento dentário 17 , com material fistuloso na área (Figura $1 \mathrm{~A})$. $\mathrm{O}$ paciente encontrava-se já com tomografia computadorizada da face, a qual demonstrava imagem sugestiva de perda de assoalho do seio maxilar direito, hipertrofia nos cornetos do lado direito, e velamento parcial, sugerindo o quadro de sinusite (Figura 1B e 1C). No momento não apresentou secreção purulenta. A manobra de Valsalva positivo confirmou a hipótese diagnóstica de fístula bucossinusal.

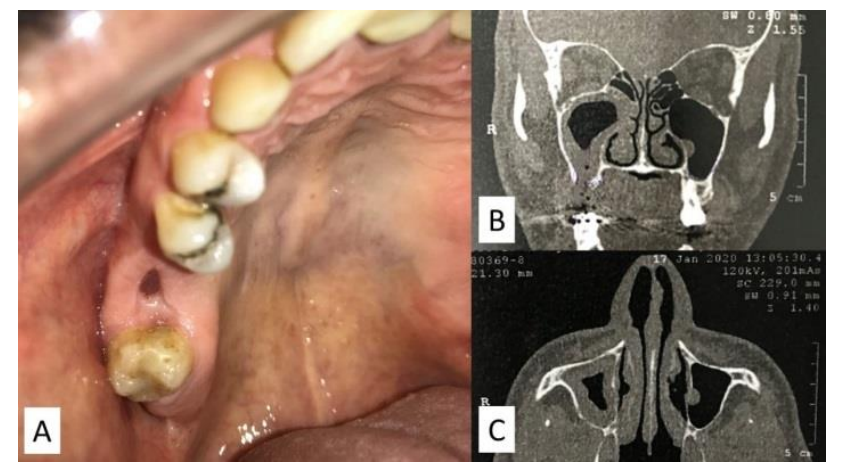

Figura 1: A) Aspecto clínico da fístula bucossinusal em maxila direita. B) Tomografia computadorizada em corte coronal demonstrando defeito ósseo em assoalho maxilar direito e C) velamento parcial.
Sendo assim, o paciente foi submetido a anestesia local (mepivacaína 3\% + epinefrina 1:200.000) em consultório odontológico. O procedimento cirúrgico iniciou-se pela realização de uma incisão trapezoidal (com duas relaxantes), descolamento subperiosteal até exposição completa da fístula (Figura 2A). Em seguida foi realizado a fistulectomia com 0 pinçamento e descolamento do tecido das paredes, evidenciando 0 real tamanho do defeito (Figura 2B).

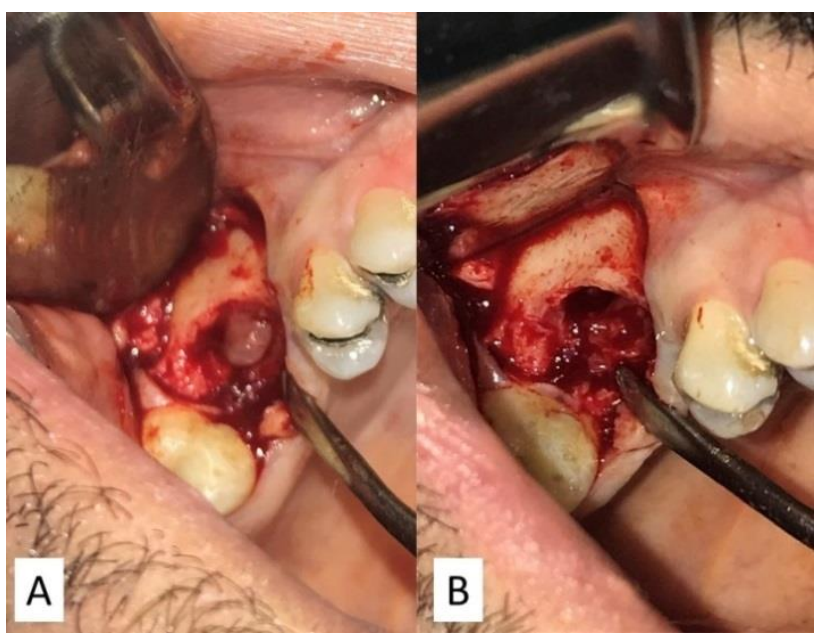

Figura 2: A) Acesso ao defeito ósseo com presença da fístula em suas paredes. B) Defeito ósseo após realização da fistulectomia.

Depois foi realizada nova incisão dentro do retalho e o divulsionamento com hemostática até a localização da gordura de Bichat, liberação do tecido, e o retalho de gordura foi avançado e reposicionado até o recobrimento completo do defeito e manutenção com sutura com fio de seda 4-0 (Figura 3A). Com uma camada de tecido gorduroso na região da fístula, seguiu-se com incisões periosteais para avanço de retalho bucal, e sutura com seda 4-0, mantendo-se assim duas camadas recobrindo 0 defeito (Figura 3B).

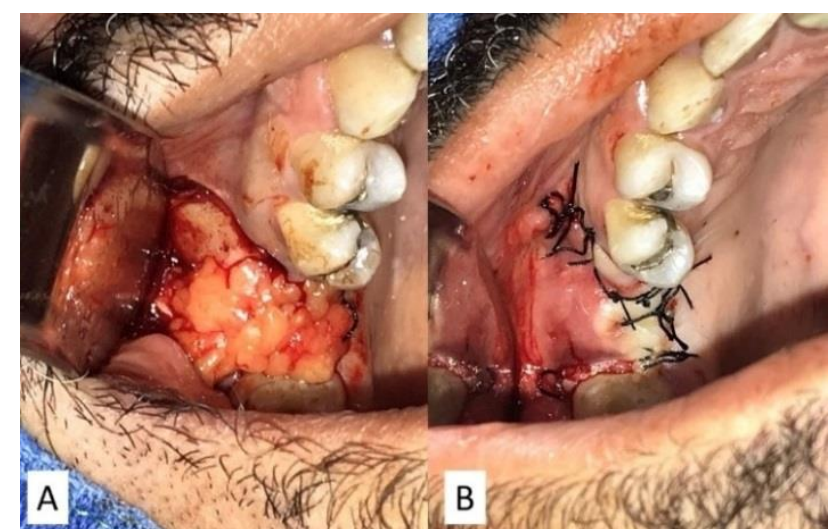

Figura 3: A) Recobrimento do defeito com avanço de retalho de gordura de Bichat. B) Sobreposição da gordura com avanço de mucosa pelo retalho bucal.

O paciente retornou com 15 dias, sem dor, sem cefaleia, sem entrada de líquidos pela região e cicatrização em normalidade (Figura 4). 


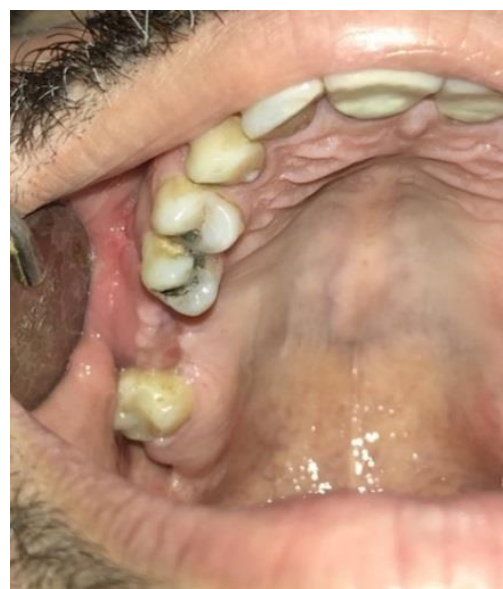

Figura 4: Aspecto clínico no $15^{\circ}$ dia de pós-operatório.

DISCUSSÃO

As principais causas relacionadas a formação de fístulas bucossinusais são associadas a patologias, traumas e extrações dentárias. As exodontias de molares superiores, apresentam maior potencial no estabelecimento de comunicações bucossinusais devido ao íntimo contato que os ápices radiculares apresentam em relação aos seios maxilares ${ }^{1,3,4}$.

São variadas as técnicas para correção de defeitos ósseos e complicações cirúrgicas, entretanto, a gordura de Bichat apresenta grandes vantagens quando comparada às demais, como: rápida epitelização, variando de 2 - 4 semanas, diferente da utilização da malha de titânio, que requer maior tempo $(6-18$ meses) para cicatrização e ainda há a necessidade de um segundo tempo cirúrgico para sua remoção; menor morbidade ao sítio doador, promovendo menor incômodo quando comparado ao retalho lingual; além de apresentar maior confiabilidade que a regeneração óssea guiada, pois estes materiais podem apresentar maior perigo, pois 0 fechamento primário das membranas é dificultoso, além destes materiais poderem ser facilmente deslocados para o seio maxilar ${ }^{1,3,4-7}$

A escolha da técnica cirúrgica utilizada para o fechamento da comunicação bucossinusal é influenciada por fatores como: quantidade e condição do tecido disponível para reparar, localização e dimensão do defeito, a presença de infecção e o tempo para diagnosticar a fístula ${ }^{6}$.

Egyedi ${ }^{8}$ afirmou que as indicações e os métodos cirúrgicos para o fechamento das comunicações bucossinusais dependem do tamanho, local e causa do defeito. Segundo ele, a utilização da gordura é indicada para comunicações que apresentam um diâmetro máximo entre $1-4 \mathrm{~cm}$, classificada, por ele, como categoria B. Sendo a categoria A, comunicações de até $1 \mathrm{~cm}$, e $B$, comunicações superiores à $4 \mathrm{~cm}$. Esta afirmação foi assegurada por este relato, em que o tecido adiposo foi utilizado para recobrimento da comunicação que apresentou aproximadamente $1 \mathrm{~cm}$ em seu diâmetro máximo.

A gordura de Bichat consiste em uma massa adiposa lobulada, com um corpo central e quatro extensões, sendo elas: bucal, pterigóidea, pterigopalatina e temporal. Esta, localiza-se em região posterior da maxila, superior as fibras do músculo bucinador.

A técnica para exposição da gordura de Bichat é considerada uma técnica simples que consiste em uma incisão vestibular em maxila, em região posterior ao pilar zigomático, com posterior incisão no periósteo e no envelope facial da gordura de Bichat. Então é realizada uma cautelosa dissecção com o auxílio de duas pinças finas, uma possibilitando a remoção da gordura e a outra para dissecar os tecidos e a mucosa oral ao redor, promovendo a exposição e o posterior reposicionamento do retalho de gordura ${ }^{6}$.

A escolha da utilização da gordura de Bichat para o recobrimento do defeito, também foi assegurada por Martín-Granizo et al. ${ }^{4}$, apresentando $96,7 \%$ de sucesso em seus casos, sendo reforçado por este relato. Em 28 de seus 29 pacientes foi notada cicatrização em normalidade, sem qualquer sinal de infecção. Apenas 1 de seus casos apresentou epitelização parcial, necessitando de um segundo tempo cirúrgico para o recobrimento com retalho mucoso para seu fechamento definitivo. Segundo o mesmo, fatores ligados ao sucesso são: cautelosa manipulação do tecido gorduroso; evitar a utilização de sugadores mecânicos, quando exposta a gordura; e, sua utilização preferível para defeitos pequenos (até $1 \mathrm{~cm})$ e médios $(1-4 \mathrm{~cm})^{8}$.

O fechamento da comunicação bucossinusal com a gordura de Bichat é uma modalidade de tratamento com altas taxas de sucesso, técnica cirúrgica relativamente fácil, baixas taxas de complicações operatórias, menor morbidade ao sítio doador, rica vascularização sanguínea, rápida epitelização e sem causar sequelas estéticas visíveis, assegurada por vários autores ${ }^{1,3,4,6-8}$ e reiterada por este relato.

CONCLUSÃO

O método utilizado, onde a aplicação clínica do corpo adiposo de Bichat foi combinada com enxerto mucoso, demonstrou ser um método simples e confiável para se obter o fechamento definitivo do defeito, sendo assegurada pela literatura e reforçada por este relato. 


\section{REFERÊNCIAS}

1. Allais $M$, Maurette PE, Cortez ALV, Laureano Filho JR, Mazzonetto R. Retalho de corpo adiposo bucal no fechamento de comunicação buco-sinusal. Rev Bras Otorrinolaringol. 2008;74(5):799-99.

2. Hanazawa $\mathrm{Y}$, Itoh $\mathrm{K}$, Mabashi $\mathrm{T}$, Sato $\mathrm{K}$. Closure of oroantral communications using a pedicled buccal fat pad graft. J Oral Maxillofac Surg. 1995;53(7):771-5; discussion 775-6.

3. Hai HK. Repair of palatal defects with unlined buccal fat pad grafts. Oral Surg Oral Med Oral Pathol. 1988;65(5):523-5.

4. Martín-Granizo R, Naval L, Costas A, Goizueta $C$, Rodriguez F, Monje F, Muñoz M, Diaz F. Use of buccal fat pad to repair intraoral defects: review of 30 cases. $\mathrm{Br} \mathrm{J}$ Oral Maxillofac Surg. 1997;35(2):81-4.

5. Procacci $P$, Alfonsi $F$, Tonelli $P$, Selvaggi $F$, Menchini Fabris GB, Borgia V, et al. Surgical treatment of oroantral communications. J Craniofac Surg. 2016;27(5):1190-96.

6. Goudar SA, Kumar R, Rathod D, Padmanabhan, Wagdargi SS. Buccal fat pad: a review. Ann Essences Dent. 2014;6(1):37.

7. Vuillemin T, Raveh J, Ramon Y. Reconstruction of the maxilla with bone grafts supported by the buccal fat pad. J Oral Maxillofac Surg. 1988;46(2):100-6.

8. Egyedi P. Utilization of the buccal fat pad for closure of oro-antral and/or oro-nasal communications. J Maxillofac Surg. 1977;5(4):241-44.

\section{CONFLITO DE INTERESSES}

Os autores declaram não haver conflitos de interesse

\section{AUTOR PARA CORRESPONDÊNCIA}

Emerson Filipe de Carvalho Nogueira

Centro Universitário Brasileiro, UNIBRA

Rua Padre Inglês, 257 - Boa Vista

50050-230 Recife - PE, Brasil

Telefone: +55 (81) 3036-0001

E-mail: emerson_filipe@hotmail.com

Submetido em 12/08/2020

Aceito em 23/10/2020 